\title{
Spin Hall effect on a noncommutative space
}

\author{
Kai Ma and Sayipjamal Dulat* \\ School of Physics Science and Technology, Xinjiang University, Urumqi, 830046, P. R. China
}

\begin{abstract}
We study the spin-orbital interaction and the spin Hall effect of an electron moving on a noncommutative space under the influence of a vector potential $\vec{A}$. On a noncommutative space we find that the commutator between the vector potential $\vec{A}$ and the electric potential $V_{1}(\vec{r})$ of lattice induces a new term which can be treated as an effective electric field, and the spin Hall conductivity obtains some correction. On a noncommutative space the spin current and spin Hall conductivity have distinct values in different direction, and depends explicitly on the noncommutative parameter. Once this spin Hall conductivity in different direction can be measured experimentally with a high accuracy, the data can be used to impose bounds on the value of the space noncommutativity parameter. We have also defined a new parameter $\varsigma=\rho \theta$ ( $\rho$ is the electron concentration, $\theta$ is the noncommutativity parameter) which can be measured experimentally. Our approach is based on the Foldy-Wouthuysen transformation which give a general Hamiltonian of a non-relativistic electron moving on a noncommutative space.
\end{abstract}

PACS numbers: 71.70.Ej, 02.40.Gh, 03.65.-w

\section{INTRODUCTION}

The approach to noncommutative quantum field theory based on star products and Seiberg-Witten maps allows for the generalization of the standard model of particle physics to the case of noncommutative space-time. Since noncommutative quantum field theory may solve the puzzles of the Standard model, there are a lot of papers concerning the quantum field theory on a noncommutative space-time [1]-[4]. Apart from these studies, many articles have been devoted to the study of various aspects of quantum mechanics (QM) on a noncommutative space (NCS) and a noncommutative phase space(NCPS), because the main goal of the noncommutative quantum mechanics (NCQM) is to find a measurable spatial noncommutativity effects. For example, the authors in Ref. [5, 6] applied NCQM to the Hydrogen atom and calculated the corrections to the Lamb shift. They find the constraint on $\theta$ is $1 / \sqrt{\theta} \geq 10^{2} \mathrm{GeV}$. Ref. [7] provided a constraint: $1 / \sqrt{\theta} \geq 3 G e V$ by studying the transitions in the Helium atom. A possibility of testing spatial noncommutativity via cold Rydberg atoms is suggested in Ref.[8]. The authors in Refs. [9]-[12] have studied the Aharonov-Bohm phase on a NCS and a NCPS. A lower bound $1 / \sqrt{\theta} \geq 10^{-6} \mathrm{GeV}$ for the space noncommutativity parameter is obtained [9]. The AharonovCasher phase for a spin-1/2 and a spin-1 particle on a NCS and a NCPS has been studied in Refs. [13]-[15]. A limit $1 / \sqrt{\theta} \geq 10^{-7} \mathrm{GeV}$ for the space noncommutativity parameter is obtained [13]. The noncommutative quantum Hall effect has been studied in Refs. [16]-[18], and Ref. [16] found a lower limit of $1 / \sqrt{\theta} \geq 10 \mathrm{GeV}$ on the noncommutativity parameter. Ref. [19] discussed noncommutative spin Hall effect (SHE) through a semi-

\footnotetext{
*Electronic address: dulat98@yahoo.com
}

classical constrained Hamiltonian, and obtained interesting results. One can use the data on the the spin Hall measurements[20] to impose some bounds on the value of the noncommutativity parameter $1 / \sqrt{\theta}$, and we find that the limit on $1 / \sqrt{\theta}$ is weaker by thirteen orders of magnitude than the one imposed by the quantum Hall effect [16]. However we propose a stronger limit on $1 / \sqrt{\theta}$ by measuring the spin Hall conductivity in different directions. The SHE is predicted by M.I. Dyakonov and V. I. Perel in 1971[21]. This effect, which occur as a result of the spin-orbit coupling between electrons and impurities, is called extrinsic. Conversely, there are intrinsic forms of the SHE, which is caused by spin-orbit coupling in the band structure of the semiconductor and survives in the limit of zero disorder, which have become an active field of research in recent years [22]-[28].

In this paper we focus on the intrinsic SHE on a NCS. To begin, we must define what we mean by "noncommutative space". NCS is a deformation of ordinary space in which the space coordinate operators $\hat{x}_{i}$ satisfy the following relation,

$$
\begin{aligned}
{\left[\hat{x}_{i}, \hat{x}_{j}\right] } & =i \theta_{i j}, \\
{\left[\hat{x}_{i}, \hat{p}_{j}\right] } & =i \hbar \delta_{i j}, \\
{\left[\hat{p}_{i}, \hat{p}_{j}\right] } & =0, \quad i, j=1,2, \cdots, n
\end{aligned}
$$

$\theta_{i j}$ is totally antisymmetric real tensor which represent the noncommutativity of the space. In addition, the product between the external fields on a NCS is,

$$
\hat{f}(\hat{\vec{x}}) \hat{g}(\hat{\vec{x}}) \equiv f(\vec{x}) * g(\vec{x})=\left.\exp \left[\frac{i \theta_{i j}}{2} \partial_{x_{i}} \partial_{y_{j}}\right] f(\vec{x}) g(\vec{y})\right|_{\vec{x}=\vec{y}}
$$

where $f(\vec{x})$ and $g(\vec{x})$ are two arbitrary infinitely differentiable functions on a commutative $R^{3+1}$ space, and $\hat{f}(\hat{\vec{x}})$ and $\hat{g}(\hat{\vec{x}})$ are the corresponding functions on a NCS. In 
NCQM one replaces the position operator $\hat{x}_{i}$ with the $\theta$-deformed

$$
\hat{x}_{i}=x_{i}+\frac{1}{\hbar} \theta_{i j} p_{j}, \quad i, j=1,2 \cdots, n
$$

and then applies the usual rules of QM. We use the following notation for the commutator of two arbitrary physical observable operators $\hat{A}$ and $\hat{B}$ in NCQM,

$$
[\hat{A}, \hat{B}]=\hat{A} \hat{B}-\hat{B} \hat{A}=[A, B]_{*}=A * B-B * A .
$$

Note that on a NCS $\hat{\vec{A}} \times \hat{\vec{A}} \neq 0$, this can be seen clearly via,

$$
\hat{\vec{A}} \times \hat{\vec{A}}=\vec{A} \times{ }_{*} \vec{A}=A_{i} * A_{j} \epsilon_{i j k} \vec{e}_{k} \neq A_{j} * A_{i} \epsilon_{i j k} \vec{e}_{k} \neq 0 .
$$

where the $\vec{A} \times{ }_{*} \vec{A}=\hat{\vec{A}} \times \hat{\vec{A}}$ indicates cross product on a NCS. Note that one of the most interesting things in noncommutative quantum field theory is that even the $U(1)$ gauge group has non-Abelian like characteristics such as self-interactions.

This paper is organized as follows: in the next section we will provide the Foldy-Wouthuysen Hamiltonian of a non-relativistic spin-1/2 particle moving on a NCS under the influence of some external electromagnetic fields. In section 3, we will calculate the spin-dependent electric current and the spin Hall conductivity. The conclusion is given in the final section.

\section{SPIN-ORBITAL INTERACTION}

In this section we will present the general form of Foldy-Wouthuysen Hamiltonian $H_{F W}$ of an electron in the presence of external fields on a NCS. As we know in the Dirac representation the wave function of a free spin-half particle is generally determined by four component spinors. And the Dirac equation has positive and negative total energy solutions. In the non-relativistic limit, we are usually only interested in the positive energy part of the spectrum that describes electrons (rather than positrons). The Foldy-Wouthuysen transformation has been proposed in Ref.[29]. Its main goals are transformation of Dirac Hamiltonians to the block-diagonal form, (it separates distinct components of relativistic spinor wave function and energy spectrum), and establishment of connection between the relativistic QM and the QM as well as the classical physics.

Let us start with the Dirac Hamiltonian for a particle with charge $e$ interacting with an external electromagnetic field,

$$
H=c \vec{\alpha} \cdot\left(\vec{p}-\frac{e}{c} \vec{A}\right)+\beta m c^{2}+e V(\vec{r})
$$

where $\beta=\gamma_{0}$ and $\alpha_{i}=\gamma_{0} \gamma_{i}$ are the Dirac matrices; $\vec{p}=-i \hbar \vec{\nabla}$ is the canonical momentum operators; $\vec{A}$ is the vector potential. The non-relativistic limit of the Dirac equation (7) is reached by a Foldy-Wouthuysen unitary transformation, which block diagonalizes the Dirac Hamiltonian by separating the positive and negative energy part of its spectrum. By a unitary transformation [30] the transformed Foldy- Wouthuysen Hamiltonian is given by,

$$
H_{F W}=\beta\left(m c^{2}+\frac{O^{2}}{2 m c^{2}}\right)+\epsilon-\frac{1}{8 m^{2} c^{4}}[O,[O, \epsilon]],
$$

where,

$$
O=c \vec{\alpha} \cdot\left(\vec{p}-\frac{e}{c} \vec{A}\right), \quad \epsilon=e V(\vec{r}) .
$$

On a NCS the Foldy-Wouthuysen Hamiltonian is,

$$
\hat{H}_{F W}=\beta\left(m c^{2}+\frac{\hat{O}^{2}}{2 m c^{2}}\right)+\hat{\epsilon}-\frac{1}{8 m^{2} c^{4}}[\hat{O},[\hat{O}, \hat{\epsilon}]] .
$$

We now calculate the various terms of $\hat{H}_{F W}$ explicitly. For the second term we have,

$$
\begin{aligned}
\hat{O}^{2}= & O * O=c^{2}\left(\vec{p}-\frac{e}{c} \vec{A}\right) *\left(\vec{p}-\frac{e}{c} \vec{A}\right) \\
& -e c \hbar \vec{\Sigma} \cdot \vec{B}+i e^{2} \vec{\Sigma} \cdot(\vec{A} \times * \vec{A})
\end{aligned}
$$

here we have used the following relations for two arbitrary vectors $\vec{A}$ and $\vec{B}$,

$$
(\vec{\alpha} \cdot \vec{A})(\vec{\alpha} \cdot \vec{B})=\vec{A} \cdot \vec{B}+i \vec{\Sigma} \cdot(\vec{A} \times \vec{B}),
$$

and,

$$
\operatorname{curl}(f \vec{A})=f \operatorname{curl} \vec{A}+\operatorname{grad} f \times \vec{A} .
$$

as well as equation(6). Next we look at the commutator $[\hat{O}, \hat{\epsilon}]$,

$$
\begin{aligned}
{[\hat{O}, \hat{\epsilon}] } & =[O, \epsilon]_{*} \\
& =-i c e \hbar \vec{\alpha} \cdot \vec{\nabla} V(\vec{r})-e^{2} \vec{\alpha} \cdot[\vec{A}, V]_{*}
\end{aligned}
$$

Furthermore, we can calculate the commutator,

$$
\begin{aligned}
{[\hat{O},[\hat{O}, \hat{\epsilon}]]=} & {\left[O,[O, \epsilon]_{*}\right]_{*} } \\
= & e c^{2} \hbar \vec{\nabla} \cdot \vec{E}+i e c^{2} \hbar^{2} \vec{\Sigma} \cdot(\vec{\nabla} \times \vec{E}) \\
& +2 e c^{2} \hbar \vec{\Sigma} \cdot(\vec{E} \times \vec{p}) \\
& -2 i e^{2} c \vec{\Sigma} \cdot\left([\vec{A}, V]_{*} \times \vec{p}\right) .
\end{aligned}
$$

In the calculations above we have neglected the higher order terms in $\theta$ such as $\left[\vec{A},[\vec{A}, V]_{*}\right]_{*}$ and $\vec{\nabla} \cdot[\vec{A}, V]_{*}$. Adding the various terms we have,

$$
\begin{aligned}
\hat{H}_{F W}= & \beta\left(m c^{2}+\frac{1}{2 m}(\vec{p}-e \vec{A} / c) *(\vec{p}-e \vec{A} / c)\right) \\
& +e V(\vec{r})-\frac{e \hbar \beta}{2 m c} \vec{\Sigma} \cdot \vec{B}-\frac{i e \hbar^{2}}{8 m^{2} c^{2}} \vec{\Sigma} \cdot(\vec{\nabla} \times \vec{E}) \\
& -\frac{e \hbar}{4 m^{2} c^{2}} \vec{\Sigma} \cdot(\vec{E} \times \vec{p})-\frac{e \hbar^{2}}{8 m^{2} c^{2}} \vec{\nabla} \cdot \vec{E} \\
& -\frac{e \hbar}{4 m^{2} c^{2}} \vec{\Sigma} \cdot(\hat{\vec{E}} \times \vec{p})-\frac{e \hbar \beta}{2 m c} \vec{\Sigma} \cdot \hat{\vec{B}}
\end{aligned}
$$


where $\hat{\vec{E}}=-\frac{i e}{c \hbar}[\vec{A}, V]_{*}$ and $\hat{\vec{B}}=-\frac{i e}{c \hbar} \vec{A} \times * \vec{A}$. The $\mathrm{FW}$ Hamiltonian (13) describes the dynamics of an electron, but the model will also equally apply to a positron ( which corresponds to a hole in solid). Now we discuss the individual terms of (13). The terms in the first parenthesis result from the expansion of $\left[(\vec{p}-e / c \vec{A})^{2}+m\right]^{1 / 2}$ and describe the relativistic mass increases, and we will neglect the corresponding noncommutative corrections in the next section. The second term describes the electrostatic energy. The third term is a magnetic dipole energy which induces Zeeman effect. The next two terms contain the spin-orbital interaction (Rashba spin-orbital coupling for the constant electric field which is along the $\vec{z}$ direction $\vec{E} \sim \vec{k}$ (unite vector)). This can be seen clearly under the assumption of a spherically symmetric potential with $\vec{\nabla} \times \vec{E}=0$, then we have,

$$
\vec{\sigma} \cdot(\vec{E} \times \vec{p})=-\frac{1}{r} \frac{\partial V}{\partial r}(\vec{\sigma} \cdot \vec{L}) .
$$

The last two terms are the noncommutative corrections of the electron moving on a NCS. For the term $\vec{\Sigma} \cdot(\hat{\vec{E}} \times \vec{p})$, explicitly, it is an effective spin-orbital interaction and the corresponding effective electric field is $\hat{\vec{E}}=-i e /(c \hbar)[\vec{A}, V(\vec{r})]_{*}$. The term $\vec{\Sigma} \cdot \hat{\vec{B}}$ is an effective Zeeman interaction, and the effective magnetic field is,

$$
\hat{\vec{B}}=-\frac{i e}{c \hbar} \vec{A} \times * \vec{A}=-\frac{i e}{c \hbar}\left(x_{2} * x_{1}-x_{1} * x_{2}\right) \vec{z}=\frac{e \theta}{c \hbar} \vec{z} .
$$

Note that the important physical quantity for the dynamics of the charged particles is the vector potential $\vec{A}$, and one can understand this via Aharonov-Bohm effect. Thus in our discussion we will impose a particular situation where $\vec{B}=\nabla \times \vec{A}$ and the Coulomb gauge $\vec{\nabla} \cdot \vec{A}=0$, where $\vec{A}=\left(x_{2}, x_{1}, 0\right)$.

\section{SPIN HALL EFFECT}

In this section we will calculate the spin-depended electric current. This is performed by incorporating spin and spin-orbital interaction into the dynamics of charge carriers as in Ref. [25]. This allows one to obtain universal expression for the spin Hall conductivity on a NCS. We must notice that for a macroscopic system the total electric potential $V(\vec{r})$ is the sum of external electric potential $V_{0}(\vec{r})$ and the lattice electric potential $V_{1}(\vec{r})$. Collecting the dynamical terms and spin-orbital coupling terms in the Hamiltonian (13) we have,

$$
\begin{aligned}
\hat{\mathcal{H}} \equiv & \frac{\vec{p}^{2}}{2 m}+\frac{e \hbar}{4 m^{2} c^{2}} \vec{\sigma} \cdot\left[\left(\vec{\nabla} V(\vec{r})-\frac{i e}{c \hbar}[\vec{A}, V]_{*}\right) \times \vec{p}\right] \\
& +e V(\vec{r}) \\
\equiv & \frac{\vec{p}^{2}}{2 m}-\frac{e \hbar}{4 m^{2} c^{2}} \vec{\sigma} \cdot(\hat{\overrightarrow{\mathcal{E}}} \times \vec{p})+e V(\vec{r}),
\end{aligned}
$$

where $\hat{\overrightarrow{\mathcal{E}}}=-\vec{\nabla} V(\vec{r})+\frac{i e}{c \hbar}[\vec{A}, V]_{*}$. By now we have derived the general formalism for spin-orbital interaction on a NCS. Heisenberg algebra for canonically conjugated variables $\vec{p}$ and $\vec{r}$ on a NCS is

$$
\begin{aligned}
\dot{\overrightarrow{\vec{r}}}= & \frac{1}{i \hbar}[\vec{r}, \hat{\mathcal{H}}]=\frac{\vec{p}}{m}+\frac{e \hbar}{4 m^{2} c^{2}} \vec{\sigma} \times \vec{\nabla} V(\vec{r}) \\
& -\frac{i e^{2}}{4 m^{2} c^{3}} \vec{\sigma} \times[\vec{A}, V]_{*}, \\
\dot{\overrightarrow{\vec{p}}}= & \frac{1}{i \hbar}[\vec{p}, \hat{\mathcal{H}}]=-e \vec{\nabla} V(\vec{r}) \\
& -\frac{e \hbar}{4 m^{2} c^{2}} \vec{\nabla}[(\vec{\sigma} \times \vec{\nabla} V(\vec{r})) \cdot \vec{p}] \\
& +\frac{i e^{2}}{4 m^{2} c^{3}} \vec{\nabla}\left[\left(\vec{\sigma} \times[\vec{A}, V]_{*}\right) \cdot \vec{p}\right] .
\end{aligned}
$$

From (17) we have,

$$
\begin{aligned}
\hat{\vec{p}}= & m \dot{\overrightarrow{\vec{r}}}-\frac{e \hbar}{4 m c^{2}} \vec{\sigma} \times \vec{\nabla} V(\vec{r}) \\
& +\frac{i e^{2}}{4 m^{2} c^{3}} \vec{\sigma} \times[\vec{A}, V]_{*}, \\
\hat{\vec{p}}= & m \ddot{\overrightarrow{\vec{r}}}-\frac{e \hbar}{4 m c^{2}}(\dot{\vec{r}} \cdot \vec{\nabla})(\vec{\sigma} \times \vec{\nabla} V(\vec{r})) \\
& +\frac{i e^{2}}{4 m^{2} c^{3}}(\dot{\vec{r}} \cdot \vec{\nabla})\left(\vec{\sigma} \times[\vec{A}, V]_{*}\right) .
\end{aligned}
$$

The second term in (19) is the cross product of the electron magnetic moment and the effective electric field on a NCS. Substitution of (19) and (20) into (18) yields the following form of the Newton's second law for charge carriers on a NCS

$$
m \ddot{\overrightarrow{\vec{r}}}=\hat{\vec{F}}(\vec{\sigma})=\vec{F}(\vec{\sigma})+\vec{F}_{\theta}(\vec{\sigma}),
$$

where the spin-dependent force $\hat{\vec{F}}(\vec{\sigma})$ on a NCS is given by,

$$
\begin{aligned}
& \vec{F}(\vec{\sigma})=-\frac{e \hbar}{4 m c^{2}} \dot{\vec{r}} \times[\vec{\nabla} \times(\vec{\sigma} \times \vec{\nabla} V(\vec{r}))]-e \vec{\nabla} V(\vec{r}), \\
& \vec{F}_{\theta}(\vec{\sigma})=\frac{i e^{2}}{4 m c^{3}} \dot{\vec{r}} \times\left[\vec{\nabla} \times\left(\vec{\sigma} \times[\vec{A}, V]_{*}\right)\right] .
\end{aligned}
$$

Here we neglected the terms proportional to $1 / c^{4}$. Note that the force in (21) is equivalent to the Lorentz force,

$$
\hat{\vec{F}}(\vec{\sigma})=\frac{e}{c}(\dot{\vec{r}} \times \hat{\vec{B}}(\vec{\sigma}))-e \vec{\nabla} V(\vec{r}) .
$$

which acts on a particle of charge $Q=e$ in the electric field $\vec{E}=-\vec{\nabla} V(\vec{r})$ and magnetic field,

$$
\hat{\vec{B}}(\vec{\sigma})=\vec{\nabla} \times \hat{\vec{A}}(\vec{\sigma})=\vec{\nabla} \times\left[\vec{A}(\vec{\sigma})+\vec{A}_{\theta}(\vec{\sigma})\right],
$$

where

$$
\begin{aligned}
\vec{A}(\vec{\sigma}) & =-\frac{\hbar}{4 m c} \vec{\sigma} \times \vec{\nabla} V(\vec{r}) \\
\vec{A}_{\theta}(\vec{\sigma}) & =\frac{i e}{4 m c^{2}} \vec{\sigma} \times[\vec{A}, V]_{*}
\end{aligned}
$$


The Hamiltonian (16) can be written as,

$$
\hat{\mathcal{H}}=\frac{1}{2 m}\left(\vec{p}-\frac{e}{c} \hat{\vec{A}}(\vec{\sigma}) \cdot\right)^{2}
$$

To solve the equation (21), the velocity relaxation time $\tau$ must be given experimentally. We assume that to the first approximation the velocity relaxation time $\tau$ of charge carriers is independent of $\vec{\sigma}$. Because of relative smallness of the spin-dependent force, we can treat $\hat{\vec{F}}(\vec{\sigma})$ in $(21)$ as a perturbation. The solution of (21) can be written in the form $\dot{\vec{r}}=\dot{\vec{r}}+\dot{\vec{r}}_{\theta}$, where $\dot{\vec{r}}$ is the $\theta$ independent solution that is given in Ref.[25], and $\dot{\vec{r}}_{\theta}$ is a small $\theta$-dependent part of the velocity which also can be obtained perturbatively. In the presence of a constant external electric field $\vec{E}=-\vec{\nabla} V_{0}(\vec{r})$, from (21), (22) and (23) we obtain,

$$
\begin{aligned}
& \langle\dot{\vec{r}}\rangle=\frac{e \tau}{m} \vec{E}-\frac{\hbar e^{2} \tau^{2}}{4 m^{3} c^{2}} \vec{E} \times\langle\vec{\nabla} \times[\vec{\sigma} \times \vec{\nabla} V(\vec{r})]\rangle, \\
& \left\langle\dot{\vec{r}}_{\theta}\right\rangle=\frac{i e^{3} \tau^{2}}{4 m^{3} c^{3}} \vec{E} \times\left\langle\vec{\nabla} \times\left(\vec{\sigma} \times[\vec{A}, V]_{*}\right)\right\rangle .
\end{aligned}
$$

Using our simple choice for vector potential $\vec{A}=$ $\left(x_{2}, x_{1}, 0\right)$, we can obtain,

$$
[\vec{A}, V]_{*}=2 i \theta \vec{\eta} \cdot \vec{\nabla} V(\vec{r})
$$

where $\vec{\eta}$ is a tensor with the following components $\eta_{11}=$ $1, \eta_{22}=-1, \eta_{12}=\eta_{21}=0$. The right-hand side of (29) and (30) contains the volume average of electrostatic crystal potential $\partial_{i} \partial_{j} V_{1}(\vec{r})$. The SHE in a cubic lattice on a commutative space has been studied in Ref. [25]. In the following we will study the noncommutative SHE in a cubic lattice. On a commutative space, for a cubic lattice, the only invariant permitted by symmetry is,

$$
\left\langle\frac{\partial^{2} V_{1}(\vec{r})}{\partial r_{i} \partial r_{j}}\right\rangle=\chi \delta_{i j},
$$

where $\chi$ is a constant which have been determined in Ref. [25]. However, on a NCS, the spacial symmetry is twisted by the noncommutativity of space coordinates through the commutator between vector potential and electric potential. This correction is represented by formalism (31) up to first order of $\theta$. Here, we are only interested in the first order correction. With the help of (31) and (32) we obtain,

$$
\left\langle\dot{\vec{r}}_{\theta}\right\rangle=\frac{e^{3} \tau^{2} \chi \theta}{m^{3} c^{3}}(\vec{\eta} \cdot \vec{\sigma} \times \vec{E}) .
$$

The density matrix of the charge carriers in the spin space can be written as,

$$
\rho^{s}=\frac{1}{2} \rho(1+\vec{\lambda} \cdot \vec{\sigma})
$$

where $\rho$ is the total concentration of charges carrying the electric current; $\vec{\lambda}$ is the vector of spin polarization of the electron fluid. The $\theta$-independent and $\theta$-dependent currents are,

$$
\begin{aligned}
& \vec{j}=e\left\langle\rho^{s} \dot{\vec{r}}\right\rangle \equiv \vec{j}^{0}+\vec{j}^{s}(\vec{\sigma}), \\
& \vec{j}^{0}=\sigma_{H} \vec{E}, \quad \vec{j}^{s}(\vec{\sigma})=\sigma_{H}^{s}(\vec{\lambda} \times \vec{E}), \\
& \vec{j}_{\theta}^{s}(\vec{\sigma})=e\left\langle\rho^{s} \dot{\vec{r}}_{\theta}\right\rangle=\sigma_{H_{\theta}}^{s}(\vec{\eta} \cdot \vec{\lambda} \times \vec{E}),
\end{aligned}
$$

where the corresponding Hall conductivities are given by,

$$
\begin{gathered}
\sigma_{H}=\frac{e^{2} \tau \rho}{m}, \\
\sigma_{H}^{s}=\frac{\hbar e^{3} \tau^{2} \rho \chi}{2 m^{3} c^{2}}, \\
\sigma_{H_{\theta}}^{s}=\frac{e^{4} \tau^{2} \chi \varsigma}{m^{3} c^{3}},
\end{gathered}
$$

where $\varsigma=\rho \theta$, and $\rho \sim 10^{22} \mathrm{~cm}^{-3}$. Thus we see that the noncommutativity parameter $\theta$ makes an important contribution to the spin Hall conductivity. Explicitly, its contribution to the spin current $\hat{\vec{j}}^{s}=\vec{j}^{s}+\vec{j}_{\theta}^{s}$ and spin Hall conductivity $\hat{\sigma}_{H}^{s}=\sigma_{H}^{s}+\sigma_{H_{\theta}}^{s}$ is order of $e \theta /(c \hbar)$. Furthermore, if the external electric field $\vec{E}$ is along the $z$-axis, the components of the spin current can be written as,

$$
\begin{gathered}
\hat{j}_{x}^{s}(\vec{\sigma})=\left(\sigma_{H}^{s}-\sigma_{H_{\theta}}^{s}\right) E \\
\hat{j}_{y}^{s}(\vec{\sigma})=-\left(\sigma_{H}^{s}+\sigma_{H_{\theta}}^{s}\right) E
\end{gathered}
$$

where $E$ is the absolute value of $\vec{E}$. Note that on a NCS, the system has distinct transport behavior in different directions, namely, the absolute spin Hall conductivity has different values in different directions, and the separation is $2 \sigma_{H_{\theta}}^{s}$. If we can measure it with sufficient precision, then we would have a clear evidence for the space noncommutativity. If $\theta=0$, our result agrees with that of Ref. [25]. Note also that since the noncommutativity of space breaks rotational symmetry, the non-trivial noncommutative correction terms of the spin Hall conductivity have a preferred direction.

\section{CONCLUSION}

The origin of SHE is the spin-orbit interaction. By using the Foldy-Wouthuysen transformation, which give a general Hamiltonian of a non-relativistic moving Dirac particle, we derived the general form of the effective spinorbital coupling on a NCS, in which the upper and lower 
two components of the electrons wave function are separated completely. Thus our formalisms are applicable both for electrons and positrons. In our investigation of the spin Hall effect, we consider a particular situation, namely, the electron is influenced by the vector potential $\vec{A}$ but the magnetic field $\vec{B}$ is absent. With this choice the first order correction of (31) is obtained in a natural way. After solving the "Newton equation" perturbatively in the framework of NCQM, we derived the spin-depended electric current whose expectation value gives the spin Hall effect and spin Hall conductivity on a NCS. We find that on a NCS the commutator between vector potential $\vec{A}$ and electric potential of lattice $V_{1}(\vec{r})$ induces a new term which can be treated as an effective electric field, and the spin Hall conductivity is corrected accordingly. In the presence of the NCS the spin Hall conductivity takes different values in different direction, and depends explicitly on the noncommutativity parameter. In addition the noncommutative correction ratio is,

$$
R=\sigma_{H_{\theta}}^{s} / \sigma_{H}^{s}=2 e \theta /(c \hbar)
$$

By using experimental data [20] of spin Hall conductivity we can impose a lower limit of $1 / \sqrt{\theta} \geq 10^{-12} \mathrm{GeV}$ on the noncommutativity parameter by setting the noncommutative correction ratio $R$ to be of order one. Although this limit on $1 / \sqrt{\theta}$ is weak, we may also impose a stronger limit on the magnitude of the noncommutativity parameter, by measuring the difference of spin Hall conductivity at different direction. However, there is no such experimental data available yet. We also have defined a new parameter $\varsigma=\rho \theta$ which may be measurable experimentally as well. The results in this paper suggest that high precision measurements in quantum mechanical systems may be able to reveal the noncommutativity of space.

Acknowledgments: This work is supported by the National Natural Science Foundation of China (10965006).
[1] S. Godfrey, M. A. Doncheski, Phys. Rev. D65, (2001) 015005.

[2] M. Haghighat, M. M. Ettefaghi, Phys. Rev. D70 (2004) 034017.

[3] A. Devoto, S. DiChiara, W. W. Repko, Phys. Rev. D72, (2005) 056006.

[4] X. Calmet, Eur. Phys. J. C50, (2007) 113.

[5] M. Chaichian, M. M. Sheikh-Jabbari and A. Tureanu, Phys. Rev. Lett. 86, (2001) 2716.

[6] T. C. Adorno, M. C. Baldiotti, M. Chaichian, D. M. Gitman and A. Tureanu, Phys. Lett. B682, (2009) 235.

[7] M. Haghighat and F. Loran, Phys. Rev. D67 (2003) 096003.

[8] J.-Z. Zhang, Phys. Rev. Lett. 93 (2004) 043002.

[9] M. Chaichian, A. Demichev, P. Presnajder, M. M. Sheikh-Jabbari, A. Tureanu, Phys. Lett. B527, (2002) 149 .

[10] M. Chaichian, A. Demichev, P. Presnajder, M.M. SheikhJabbari, A. Tureanu, Nucl. Phys. B611, (2001) 383.

[11] H. Falomir, J. Gamboa, M. Loewe, F. Méndez, J. C. Rojas, Phys. Rev. D66, (2002) 045018.

[12] K. Li, S. Dulat, Eur. Phys. J. C46, (2006) 825.

[13] B. Mirza, M. Zarei, Eur. Phys. J. C32, (2004) 583.

[14] K. Li, J.-H. Wang, Eur. Phys. J. C50, (2007) 1007.

[15] B. Mirza, R. Narimani, M. Zarei, Eur. Phys. J. C48, (2006)641; S. Dulat, K. Li, Eur. Phys. J. C54, (2008) 333.

[16] B. Harms, O. Micu, J. Phys. A40, (2007) 10337.

[17] O. F. Dayi, A. Jellal, J. Math. Phys. 43, (2002) 4592.(Erratum-ibid. 45, (2004) 827; A. Kokado, T. Okamura, and T. Saito, Prog. Theor. Phys. 110, (2003) 975;
S. Dulat, K. Li, Eur. Phys. J. C60, (2009) 163.

[18] B. Chakraborty, S. Gangopadhyay, A. Saha, Phys. Rev. D70, (2004) 107707; F. G. Scholtz, B. Chakraborty, S. Gangopadhyay, A. G. Hazra, Phys. Rev. D71, (2005) 085005; F. G. Scholtz, B. Chakraborty, S. Gangopadhyay, J. Govaerts, J. Phys. A38, (2005) 9849.

[19] O. F. Dayi, M. Elbistan, Phys. Lett. A373, (2009) 131.

[20] S.O. Valenzuela, M. Tinkham, Nature, 442, (2006) 176.

[21] M. I. Dyakonov and V. I. Perel, Phys. Lett. A35,(1971) 459.

[22] X.-J. Liu, X. Liu, L. C. Kwek, C. H. Oh, Phys. Rev. Lett. 98, (2007) 026602.

[23] J. Shibata, H. Kohno, Phys. Rev. Lett. 102, (2009) 086603.

[24] M. Gradhand, D. V. Fedorov, P. Zahn, I. Mertig, Phys. Rev. Lett. 104, (2010) 186403.

[25] E. M. Chudnovsky, Phys. Rev. Lett. 99, (2007) 206601; V. Ya. Kravchenko, Phys. Rev. Lett. 100, (2008) 199703; E. M. Chudnovsky, Phys. Rev. Lett. 100, (2008) 199704;

[26] S. Murakami, N. Nagaosa, S.-C. Zhang, Science, 301, (2003) 1348;

[27] S. Murakami, N. Nagaosa, S.-C. Zhang, Phys. Rev. B69, (2004) 235206.

[28] J. Sinova, D. Culcer, Q. Niu, N. A. Sinitsyn, T. Jungwirth, A. H. MacDonald, Phys. Rev. Lett. 92, (2004) 126603.

[29] L. L. Foldy and S. Wouthuysen, Phys. Rev. 78,(1950) 29.

[30] W. Greiner, Relativistic Quamtum Mechanics: Wave Equation (Springer, 2000) 277. 